\title{
Vascular Surgery and the General Surgeon: Review Article
}

\author{
Abdulsalam Y. Taha \\ Department of Cardiothoracic and Vascular Surgery, School of Medicine, Faculty of Medical Sciences, \\ University of Sulaimaniyah and Sulaimaniyah Teaching Hospital, Sulaimaniyah, Iraq \\ Email: salamyt 1963@hotmail.com
}

Received 26 February 2015; accepted 14 March 2015; published 19 March 2015

Copyright (C) 2015 by author and Scientific Research Publishing Inc.

This work is licensed under the Creative Commons Attribution International License (CC BY).

http://creativecommons.org/licenses/by/4.0/

(c) (i) Open Access

\section{Abstract}

The vascular surgeon is trained in the management of diseases affecting all parts of the vascular system except that of the heart and brain whereas cardiothoracic surgeons manage surgical diseases of the heart and its vessels. Although vascular surgery is previously a field within general surgery, it is now considered a specialty on its own right in many countries such as the UK and the United States. Other countries such as Iraq have a mixed practice in which the cardiac or thoracic surgeon performs vascular surgery. Programs of training in vascular surgery are slightly different depending on the region of the world one is in. In the United States, a 5-year general surgery residency is followed by 2 years training in vascular surgery. In Iraq, the time table allocated for the general surgical trainee in vascular surgery is unfortunately short (1 - 3 months). This period is hardly enough for the candidate to grasp the decision-making and technical skills of vascular surgery. We believe that general surgeons need to have adequate training and expertise in vascular surgery particularly in areas and situations lacking this facility to deal with the life- and/or limbthreatening emergencies. This review article aims to orient the general surgical trainee about the scope of vascular surgery and enable them to correctly diagnose and treat common vascular emergencies such as extremity and abdominal vascular injuries (AVI).

\section{Keywords}

Vascular Surgery, General Surgeon, Extremity, Abdominal, Vascular Injuries

\section{Introduction}

Vascular surgery is a well established subspecialty for many decades. Although it was previously considered a field within general surgery, vascular surgery is now considered a specialty in its own right [1]. The specialty 
evolved from general and cardiac surgery [1]. Early leaders of the field included Russian surgeon Nikolai Korotkov [1874-1920], noted for developing early surgical techniques and is a pioneer of $20^{\text {th }}$ century vascular surgery [1] [2], American interventional radiologist Charles Theodore Dotter who is credited with inventing minimally invasive angioplasty, and Australian Robert Paton, who helped the field achieves recognition as a specialty. Edwin Wylie of San Francisco was one of the early American pioneers who developed and fostered advanced training in vascular surgery and pushed for its recognition as a specialty in the United States in the 1970s [1].

In many countries, UK for example, vascular surgery is now practiced separately from general, thoracic and cardiac surgery [3]. In other countries, vascular surgery is part of the practice of cardiac surgery (cardiovascular) or thoracic surgery (thoracic and vascular) or both cardiothoracic and vascular surgery. In Iraq, vascular surgery is practiced by a thoracic or cardiac surgeon (mixed practice).

Before the emergence of cardiothoracic surgery in Iraq, vascular surgery was practiced by other surgeons mainly general and sometimes orthopedic surgeons. And even after the beginning of cardiothoracic surgery, vascular surgery continued to be practiced by interested general surgeons in areas that lack the facility of cardiothoracic surgery.

In Iraq, most of the cardiothoracic and vascular surgeons are graduates of the Iraqi Board for Medical Specializations (IBMS) that was established in 1988 whereas minority has a high Diploma in thoracic and vascular surgery. From 1992 to 2012, there were 149 graduates of IBMS in cardiothoracic and vascular surgery. Unfortunately, many graduates have left the country due to lack of security while some of them were killed. Hence, the number of active vascular surgeons in Iraq is small compared to its population (estimated to be 31,234,000 in 2009). In 2008, the ratio of active thoracic surgeons per 100,000 Iraqi populations was 0.47; much lower than the American ratio of 1.36. This calculated ratio is based on all the graduates and does not take into consideration the number of surgeons who were either killed or left the country for one reason or another. Moreover, we have to take into account that the remaining surgeons unlike their colleagues in other countries are not fully committed to vascular surgery as they have an additional practice in thoracic and/or cardiac surgery.

In our opinion, the general as well as the orthopedic surgeon need to have an adequate training and a good expertise in vascular surgery particularly in areas and situations lacking the facility of proper vascular surgery to deal with emergency cases of vascular trauma in the extremities and/or the abdomen to save the life and limbs of patients [4].

\section{The Scope of Vascular Surgery}

Vascular surgery is a specialty of surgery in which diseases of the vascular system (arteries, veins, and lymphatics) are managed by medical therapy, minimally-invasive catheter procedures, and surgical reconstruction [1] [3]. Among the main procedures undertaken by vascular surgeons are carotid endarterectomy, endovascular aneurysm repair and thrombectomy [3]. As many as 50 per cent of patients with vascular disease present urgently or as an emergency, and in the past have often been managed by a general surgeon [3]. In Iraq, vascular surgeons deal with a wide spectrum of emergent and elective conditions such as vascular trauma in different parts of the body, thromboembolectomy, vascular access for hemodialysis, peripheral arterial disease, varicose veins, abdominal aortic occlusion and aneurysm, carotid endarterectomy, dorsal and lumbar sympathectomy and others.

\section{Vascular Surgery Training}

Programs of training in vascular surgery are slightly different depending on the region of the world one is in [1]. In the United States, there are two pathways; the traditional path involves a five year general surgery residency followed by a 1 - 2 year (typically 2 years) vascular surgery fellowship. An alternative path is to perform a five or six year vascular surgery residency [1]. In Iraq, postgraduate students in general surgery as well as some other specialties such as orthopedics, plastic and pediatric surgery are exposed to vascular surgery training for a relatively short time (1 - 3 months). This period is hardly enough for the candidate to grasp the decision-making and technical skills of vascular surgery.

Therefore; there is a need to focus on the common emergencies such as acute limb ischemia due to vascular trauma as well as abdominal vascular injuries (AVIs). It is crucial for any trauma surgeon to be able to make an early diagnosis of vascular injuries on clinical bases, order and use bed-side procedures such as a portable Doppler examination, start an effective initial management to stop bleeding, treat shock and stabilize fractures 
and/or dislocations and embark on definitive management to repair the vessels or if not capable to do so; refer the patient as soon as possible to a vascular surgical center to have a timely limb revascularization and thus prevent limb loss.

\section{Extremity Vascular Injuries (EVIs)}

Vascular trauma of the limbs is a common emergency both in civil and war time. It accounts for approximately $1 \%-3 \%$ of total civilian trauma in various studies [5]. With the worldwide rise in the rate of terrorist attacks and high speed motor vehicle crash, the rate of vascular trauma has increased as well [5]. Our hospitals throughout the country daily receive such casualties and all surgeons should get the necessary knowledge and skill to make an early diagnosis and initiate a timely therapy. The management of such injuries is a challenging job for general surgeons, particularly in developing countries, where limited resources make this task even more difficult [5].

\subsection{Etiology}

Extremity vascular injuries can result from blunt and penetrating mechanisms. The penetrating agents may be a knife, sharp object, bullet, pellet, shrapnel... Blunt trauma can follow a road traffic accident, falling from a height... Beware of the association between fractures and/or dislocations and vascular injuries. Examples include posterior knee dislocation and popliteal artery injury, supracondylar humeral fracture and brachial artery injury. Iatrogenic vascular injuries are increasingly encountered with the increase in conventional peripheral angiography and cardiac catheterization [6].

\subsection{Pathophysiology}

The trauma can involve arteries, veins or both. Venous injuries can bleed profusely and may be complicated by air embolism particularly when the central veins are involved. Moreover, the thin wall of the vein renders them difficult to handle, therefore, the seriousness of vein injuries should not be under-estimated [6].

Arterial injuries are classified into total transaction, partial tear, arterial contusion, false or pseudoaneurysm and arterio-venous fistula (AVF) (Figures 1(A)-(G)). External bleeding results from complete and partial tear. On the other hand, there is no bleeding from arterial contusion or AVF. Hence, absence of bleeding does not exclude a vascular injury. In complete arterial cut, there is retraction, vasoconstriction and thrombosis which can

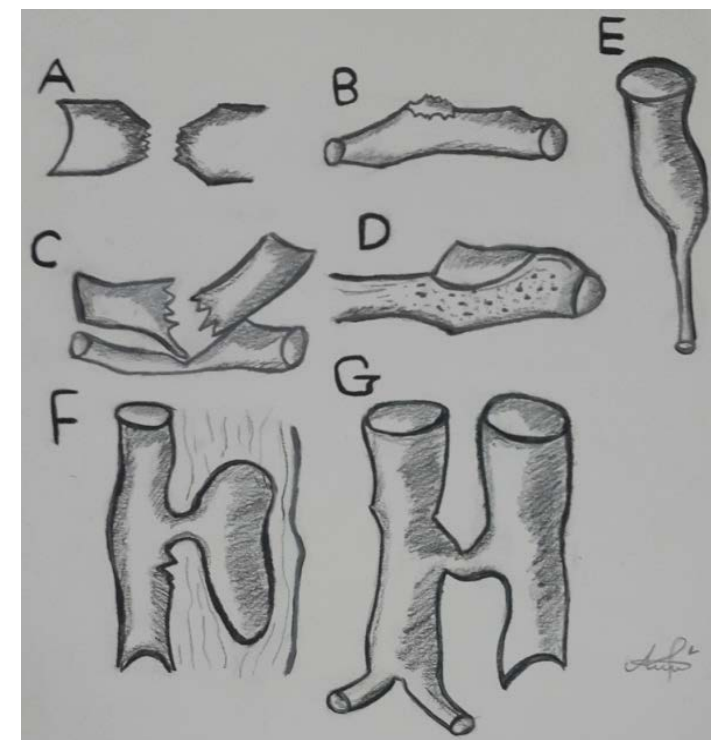

Figure 1. Types of arterial injuries. (A) Complete transaction; (B) Laceration; (C) Arterial compression; (D) Arterial contusion; (E) Contusion and spasm; (F) False aneurysm; (G) AVF. 
arrest the bleeding after a while. In contrast, vessel retraction in a lateral arterial tear increases the size of the rent and the amount of bleeding. One sequel of lateral tear is the formation of pseudoaneurysm (pulsating hematoma). This results from local bleeding into the nearby soft tissues (local hematoma). The outer wall of the hematoma gets organized over time forming a fibrous wall while blood flow continues through the artery and preserves the distal pulses. In arterial contusion, there is an intimal damage, subintimal hematoma and local thrombus formation. The blood flow ceases although there is no arterial discontinuity. The site of contusion is darkly discoloured with a bounding proximal and absent distal pulse. In AVF, an adjacent artery and vein are injured resulting in a short-circuiting of blood from the artery to the vein. In this serious injury, there is no bleeding and the blood flow is not interrupted and hence the limb viability is not affected. However, there are 2 cardinal signs of this injury, namely thrill and bruit which should be sought by the examining doctor; otherwise AVF can be easily missed. Arterial spasm may result from compression of the artery by a piece of fractured bone and usually resolves with fracture reduction. It should not be mixed with arterial contusion that can similarly follow bone compression but has a different pathology and treatment. Arterial contusion is a real damage that requires resection of the damaged segment and end to end anastomosis whereas arterial spasm is usually managed conservatively with a local application of a vasodilator agent such as papaverine and sometimes by using a Fogarty catheter for dilation [6].

\subsection{Clinical Features}

- Understanding the pathology of different types of arterial injury is important to know the symptoms and signs. Interruption of arterial blood flow results in acute limb ischemia that is manifested by 6 Ps (pain, pallor, pulselessness, paresthesia, paralysis and poikilothermia). Paralysis and paresthesia are ominous signs as they indicate ischemia of skeletal muscles and somatic nerves; both are the most tolerant tissues to ischemia. Loss of muscle softness (woody limb) is a bad sign indicating an irreversible muscle necrosis.

- The time interval between injury and revascularization of injured limb is an important factor in determining the outcome of repair [5]. Rapid evacuation of the injured patients was the key factor in the successful management of vascular injuries in the Korean and Vietnam military conflicts [5]. A warm limb ischemic time of less than 6 hours was associated with an amputation rate of 6.7\% compared to $33 \%$ in limbs with warm ischemia of more than 6 hours in one series [O, Sullivan and Hehir cited in 4]. However, this "golden" time is not rigid; it is influenced by many factors such as the site of arterial injury, presence of good collaterals, ambient temperature, associated injuries to veins, nerves and bones... The prerequisite for repairing limb arterial injuries is limb viability; a viable limb should undergo revascularization regardless the time factor, while a dead limb should not be revascularized [4].

- Acute limb ischemia is considered to exist when Doppler signal in the extremity is absent on multiple attempts after resuscitation, warming and reduction of fractures. The signs of acute vascular injury are classified into hard and soft signs [6]. Hard signs are: pulsatile bleeding, expanding hematoma, absent distal pulses, cold pale limb, palpable thrill and audible bruit. Soft signs are small hematoma, history of hemorrhage at scene, unexplained hypotension and peripheral nerve deficit [6]. Presence of hard signs of arterial injury is considered an indication of surgery without any investigation [5].

- The state of the pulse deserves elaboration; a normal pulse may exist despite a significant vascular injury [5] such as an AVF, false aneurysm, lateral arterial tear, venous injury, an injury to a branch rather than the main artery and the presence of efficient collaterals. Moreover, a pulse wave may travel through a soft thrombus producing a distal pulse. Majid et al. [7] reported 25\% rate of palpable pulses with vascular injuries. In contrast, absent pulse may be due to anatomical variation or shock state rather than an arterial injury.

\subsection{Initial Management}

Although significant vascular injuries may present with no external bleeding, arterial and/or venous hemorrhage is an alarming and serious presentation of most acute vascular injuries. Injury to the common femoral artery is often fatal because hemorrhage control in the field is difficult. Haemostasis can be achieved by digital pressure over the proximal artery, pressure dressing, tourniquet and clamping of visible bleeders by a vascular clamp if this is feasible. When a tourniquet is used be cautious of its disadvantages such as increasing venous bleeding, nerve injuries and missed tourniquet (always report the time of its application). When these measures fail to stop the bleeding, operative control is necessary. Associated limb fractures should be stabilized and shock and other 
bodily injuries if present should be dealt with accordingly.

\subsection{Diagnosis}

The diagnosis of acute vascular injuries is straightforward in most cases due to the presence of hard or soft signs mentioned before. Frykberg et al. found that accurate physical examination was highly sensitive and specific in the diagnosis with a negative predictive value of 99\% [8]. It has also been described as the safest diagnostic approach by others [7] [9]. Initial physical examination is normal in $15 \%$ of cases with vascular injury [O, Sullivan and Hehir cited in 4]. Repeated physical examination is essential if the injury is not to be missed [4].

However, significant vascular injuries may present with viable limbs, intact distal pulses and no bleeding (AVF and false aneurysm). Therefore, do not forget to put your hand on the site of injury feeling for a thrill and put your stethoscope listening to a bruit.

Fractures and/or dislocations may be problematic! Always ask yourself is there an associated vascular injury? If doubt still exists after reducing the fracture and/or the dislocation, then Doppler ultrasonography and/or angiography are necessary to make the diagnosis. If these two techniques are not available or inconclusive, then surgical exploration is mandatory and should not be unnecessarily delayed. A negative surgical exploration is by far much better and safer than missing a vascular injury and losing a limb.

\subsection{Work-Up}

- Work-up may be needed in doubtful cases. A portable Doppler device can be used to detect a pulse wave when pulse palpation is uncertain. Hussein et al used Doppler ultrasonography and pressure studies in his doubtful cases, because of non-availability of angiography in Emergency room of their hospital [5]. The injured extremity index is similar to the ankle-brachial index and is calculated using a manual blood pressure cuff and a continuous wave Doppler. First step is to determine the pressure at which the arterial Doppler signal occludes in the injured extremity (numerator). Cuff and Doppler moved to uninjured extremity and occlusion pressure of Doppler signals recorded (denominator). Injured extremity index $>0.90$ is normal and has a high specificity for excluding extremity vascular injury in the absence of hard signs [10].

- Recently duplex ultrasonography has emerged as a valuable diagnostic tool for the diagnosis of potential vascular injuries [5]. Bynoe et al. [11] demonstrated its sensitivity and specificity of 95\% and 99\% respectively in identifying the vascular injuries. However, it is operator dependent and has a limited role in extensive soft tissue injuries and large hematoma [5].

- Arteriography is very helpful in doubtful cases providing the patient is hemodynamically stable and it does not delay the repair. Carrillo et al. [12] and Bowley et al. [13] advocated angiography only in stable patients, to delineate the site, nature and extent of injury in cases of multiple pellet injury or multiple fractures [10] [12] [13]. Extremity vasoconstriction with shock and hypothermia in young troops may lead to confusing or false-positive findings of angiography [10].

- Meissner et al. [14] have recommended a combination of physical examination, Doppler arterial pressure measurement and duplex ultrasonography as an optimum screening method for potential vascular injury.

\subsection{Exposure of Blood Vessels}

Anatomical knowledge of the vascular system is essential. For every vessel in the body (neck, thorax, abdomen, pelvis and limbs) there is one or more approach. Familiarity of the surgeon with these approaches is very important. Faced with a bleeding from a wound in the neck, axilla, groin and etc., the surgeon most unlikely has time to go and read about the most suitable approach to isolate and fix the injured vessel. Instead, he should know and immediately go through the standard approach. Otherwise, more time and blood would be lost if a wrong approach is chosen and ultimately the patient may be lost as well. Such a detailed knowledge of vascular exposure can be found in formal texts of vascular surgery and lectures [15].

\subsection{Surgical Tips}

- A general surgical set together with few vascular clamps, dissecting scissors, vascular bens and a right-angle forceps is fair enough to carry out the vascular repair. Other important tools are the Fogarty catheter, Polypropylene sutures (5-0 and 6-0 are suitable for most limb vessels) and heparinized saline (Figure 2). 

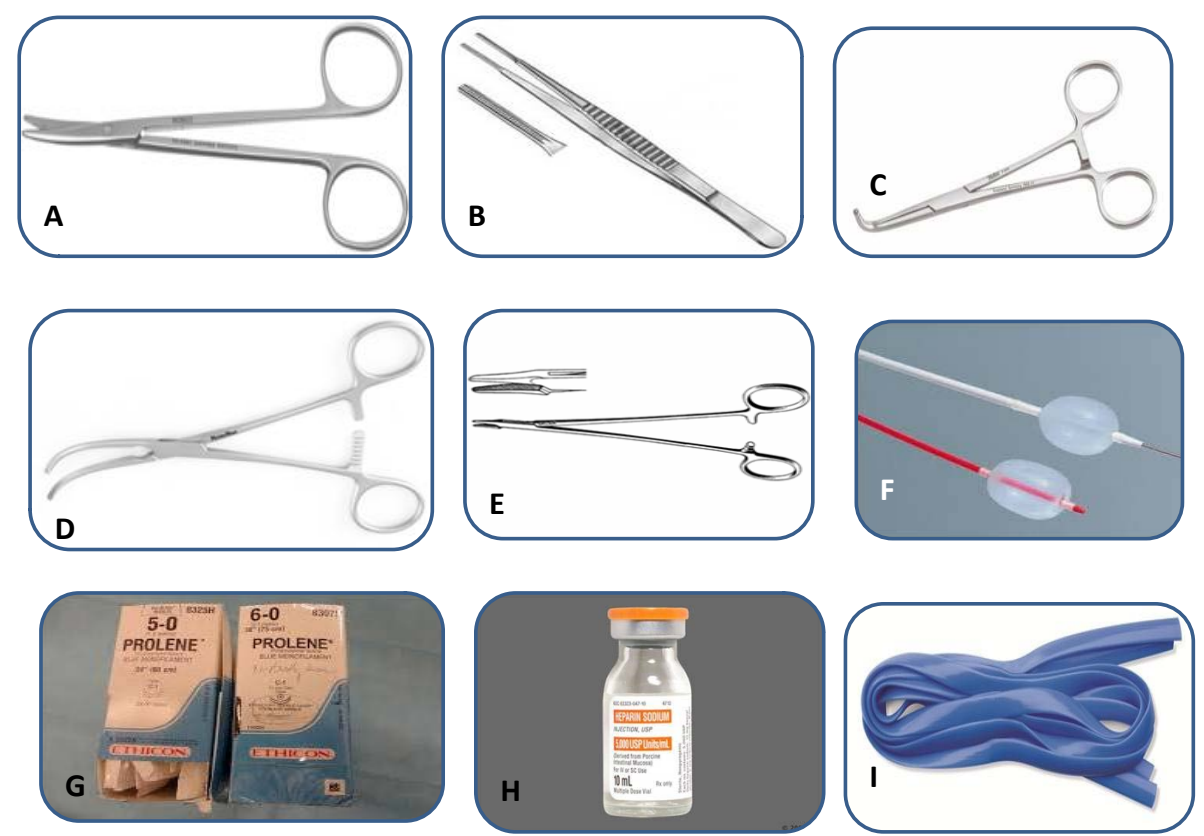

Figure 2. Essential tools for vascular repair. (A) Dissecting scissors, (B) Vascular bens, (C) Right-angle forceps, (D) Vascular clamp, (E) Vascular needle holder, (F) Fogarty catheters, (G) Polypropylene sutures, (H) Heparin, (I) Vessel loops.

- After choosing the approach, proximal and distal control of the injured vessel should be accomplished quickly so that vascular clamps are applied to stop the bleeding. Sharp dissection is done in the perivascular plane using a good pair of scissors and atraumatic vascular bens. The adventitia should be cleared from the artery and the damaged arterial tissue should be excised to finish with completely healthy arterial ends. Fogarty catheter of proper size ( $6 \mathrm{~F}$ for lower limb and $3 \mathrm{~F}$ or $4 \mathrm{~F}$ for upper limb) is passed proximally to dilate the artery and get a good antegrade flow [16]. The distal arterial segment is usually retracted and vasoconstricted and may be difficult to be found. Fogarty catheter is passed distally with the balloon deflated as far as we can to retrieve any clots by gradually inflating the balloon and pulling the catheter. This process should be repeated till we get good back bleeding. Avoid damaging the endothelium by balloon hyperinflation. The goal is clot, not intima, removal; so, do not overinflate or "drag" too much [10]. Irrigation of the distal arterial segment by heparinized saline (5000 IU UFH in 500 cc NS) is important to open up the distal circulation (regional anticoagulation) [10].

- Temporary arterial shunting has been advocated for complex wounds, for which fracture fixation and extensive debridement is required [5]. It may reduce the total ischemic time, amputations and hospitalization [5]. However, it requires more dissection and longer operative time [5].

- If the gap in the artery is less than $3 \mathrm{~cm}$, tension-free end to end anastomosis is possible (Figure 3). In doing the arterial anastomosis, it is important to gently handle the vessel. Size discrepancy of the vessels can be overcome by the technique of spatulation [15].

- However, if there is a greater loss, grafting is necessary (the best is the great saphenous vein of the uninjured limb) (Figure 4). Nearly always in the setting of trauma, the vein appears in situ as "too small" or "not adequate" due to vasoconstriction or spasm. Best assessed after hydrodistention [10]. We do not prefer the use the synthetic grafts due to financial constraints and presence of badly contaminated wounds in majority of our patients. However, some authors have reported that, the patency and infection rates of autogenous vein graft repair and PTFE graft repair are comparable [Degiannis et al. and Feliciano et al. cited in 5]. Prosthetic conduit is acceptable as a last resort in extremities when vein cannot be harvested [10].

- Venous injuries alone or in association with arterial injuries are better repaired [5]. However, badly-injured veins in a hemodynamically unstable patient can be safely ligated [5]. The resultant oedema usually resolves with an elastic support and limb elevation [5]. Repair of extremity venous injury should only be considered in stable patients [10]. Extremity veins that can be ligated routinely are brachiocephalic, subclavians, and 


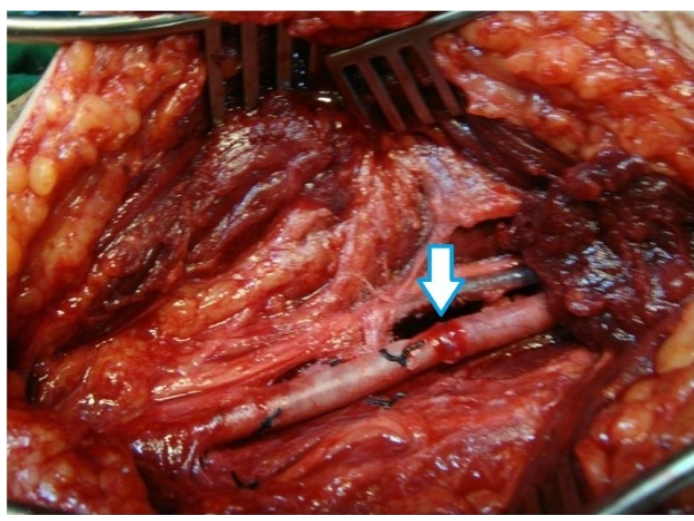

Figure 3. End to end arterial repair.

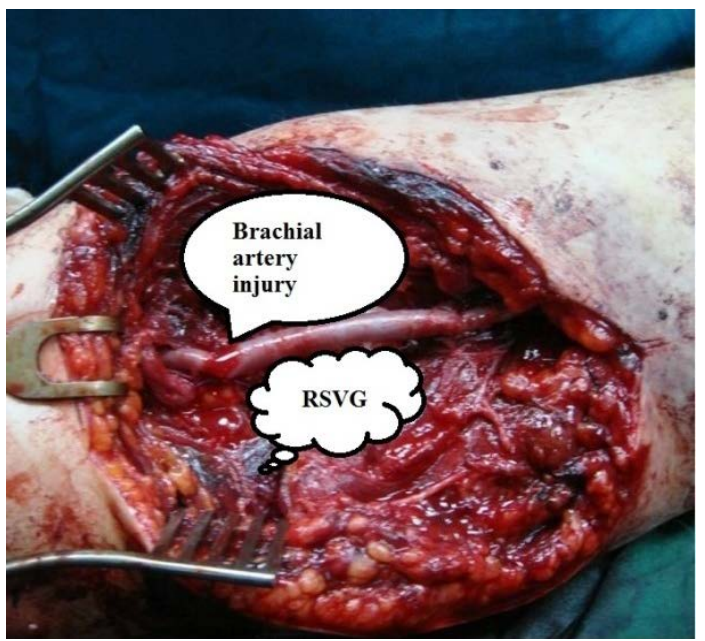

Figure 4. Interposition vein graft.

tibials [10]. The internal and external jugular, the infrahepatic inferior vena cava, left renal, mesenteric, and internal iliac veins can be similarly managed [10].

- Fasciotomy is a very important adjuvant operation in vascular repair and it should be used liberally [5]. The main indications are compartment syndrome, extensive soft tissue damage, combined arterial and venous injuries, venous ligation and following repair of late-presented arterial injuries. Among many techniques of fasciotomy, the open approach is preferred as it enables the surgeon to identify the viable tissues (healthylooking muscles that bleed and contract on cutting and stimulation) and excise the non-viable tissues (Figure 5). Prophylactic distal fasciotomies are to be considered in all patients with prolonged ischemia times [10].

- Vascular injuries associated with fractures need repair of the vessels and reduction and fixation of the fracture. The sequence of repair (vessel or bone first) depends on the individual case. If the patient presents late, the priority is for vessel repair. Otherwise, the fracture can be managed first followed by vascular repair.

- Repair of arterial injury is contra-indicated when the limb is frankly dead. Such a limb needs a primary amputation i.e. amputation without repair. This is in contradistinction to secondary amputation which follows unsuccessful vascular repair. It is very important to diagnose a dead limb and avoid its revascularization as this would result in acute tubular necrosis subsequent to myoglobin effect on the kidney.

- Primary amputation or ligation is an acceptable damage control technique when other life-threatening injuries are present [10]. This is especially true for small, more distal arteries and veins [4] [5] [10]; otherwise major limb arteries should be repaired [4]. Limb arteries that can be ligated routinely are the digital, radial or ulnar, but not both; preserve ulnar when possible, brachial distal to profundi with adequate Doppler signal at wrist, profunda femoris and branches of subclavian [10]. Likewise, the external carotid, hepatic and internal iliac arteries can be dealt with similarly [10]. 


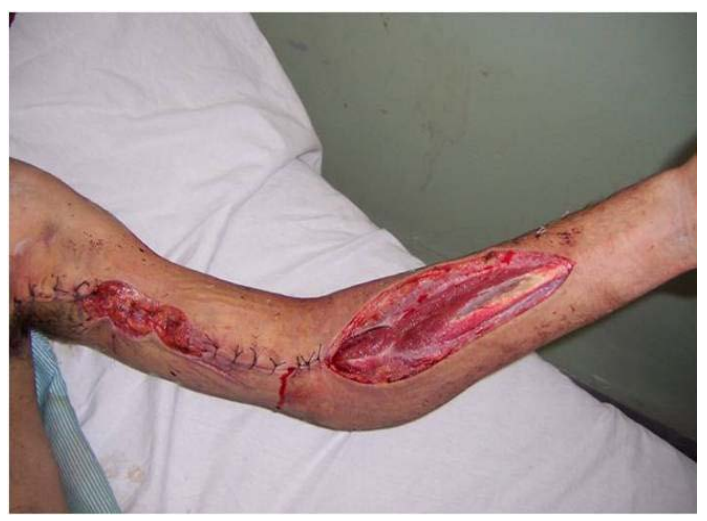

Figure 5. Fasciotomy of the forearm and partial wound closure following brachial artery repair. The patient sustained a road traffic accident and presented with profuse bleeding from the upper arm wound and features of distal limb ischaemia. Fasciotomy was performed just after vessel control. The forearm muscles proved viable; therefore, vascular repair was decided.

- Mangled extremity refers to a severely injured limb for which attempted salvage would be futile and a primary amputation is a potential outcome. Mangled Extremity Severity Score (MESS) is one scoring system used to identify such limbs and anticipate their prognosis [5]. The choice between primary and secondary amputation in vascular trauma in the extremity may be difficult [4]. Heroic attempts to save the badly-injured limb may not succeed. On the contrary, it may end in secondary amputation and/or the death of the patient [4]. If the surgeon decides on limb salvage, he should anticipate prolonged hospitalization, increased rate of sepsis, the possibility of the patient's attachment to the limb, and the possibility of poor functional outcome [4]. Primary amputation may be life-saving [4].

- Wound debridement is an essential step (all devitalized tissues should be excised). The arterial repair should be covered by viable tissue. A drain is advised after securing the hemostasis. Complete primary closure of the wounds is carried out in the very clean cases only, otherwise partial closure is preferred as the wounds are usually contaminated and carry the risk of infection.

\subsection{Postoperative Surveillance}

Bleeding and thrombosis of repair are the 2 potential postoperative complications. The most gratifying result after an arterial repair is to feel a strong distal pulse. Palpable pulses obtained in the operating room should remain palpable post-operatively [10]. Pulse changes, even if Doppler signals remain, may indicate graft thrombosis and should be investigated [10]. Portable Doppler device is useful for this purpose. The patient may need re-exploration for thrombectomy or bleeding.

\subsection{Outcome}

The outcome of vascular repair in extremity vascular trauma is measured by limb salvage rate. Until World War II, arterial ligation was the mainstay of treatment with an amputation rate of $49 \%$. On account of continuous improvements in trauma care system and formal vascular repair, amputation rate declined to $13 \%$ in Korean and Vietnam conflict. At present, these injuries are associated with amputation rate of less than $10 \%$ of the cases [5]. Porter et al. [17] observed that general surgeons with trauma experience can provide an effective treatment of peripheral vascular injuries. This observation is substantiated by the study of Hussein et al. from Pakistan in which 93 patients underwent different surgical procedures for extremity vascular trauma over a 6-year period. The patients were all managed by general surgeons as their hospital had no vascular surgery facility. Despite that, vascular reconstruction was successful in $89.3 \%$ of the patients [5]. This limb salvage rate was close to that achieved by Taha, a vascular surgeon (94.6\%) [4]. 


\section{Abdominal Vascular Injuries (AVIs)}

The general surgeon is frequently involved in the management of abdominal trauma. Alone or as a member of the trauma team, the general surgeon should always be prepared to correctly diagnose and treat abdominal vascular injuries (AVIs). One of the common scenarios in our surgical practice is to perform an exploratory laparotomy for a patient with acute abdomen due to trauma and unexpectedly discover a major bleeding and/or retroperitoneal hematoma (RPH) with or without other injuries; a situation that necessitates the help of the vascular surgeon. Meanwhile, the hemodynamic state of the patient usually gets worse due to uncontrolled hemorrhage awaiting the arrival of the vascular surgeon. The purpose of this mini-monograph is to enable the general surgeon to make a clinical diagnosis or at least to suspect AVIs preoperatively so that surgical exploration is performed in optimum operating conditions with a vascular surgeon and vascular instruments available to ensure patient's recovery in these technically challenging injuries.

\subsection{History}

DeBakey and Simeone (1946) reported 49 AVIs out of 2,471 arterial injuries (2\%) during WWII [18]. Rich et al. (1970) reported 29 AVIs out of 1000 arterial injuries (2.9\%) during Vietnam War [19]. Clouse et al. (2007) reported 18 AVIs out of 301 vascular injuries (6\%) during Operation Iraqi Liberation [20]. The incidence of AVIs in military conflicts is, therefore rising. Likewise, the improvement in pre-hospital resuscitation and care had resulted in more AVIs due to penetrating agents being encountered in civil practice as well [21].

\subsection{Epidemiology}

Up to one fourth of patients with abdominal trauma may have a major vascular injury (arteries and veins are equally involved). AVIs are mostly (up to 95\%) caused by penetrating trauma. Aortic injury is most common, occurring in one fourth of patients while the inferior vena cava (IVC) is the vein most often involved, representing approximately one third of patients with vascular injury of the abdomen [21].

\subsection{Etiology}

All vessels in the abdomen are susceptible to injury with penetrating trauma [22]. Vascular injuries in blunt trauma are far less common and usually involve the renal vessels, though all other vessels, including the aorta, can be injured. Blunt trauma results from deceleration, anteroposterior compression or pelvic fractures [22].

\subsection{Fatality}

In penetrating trauma, fifteen \% die before any surgery can be attempted. Overall mortality is greater than $50 \%$ [21]. Death can be immediate, because of hemorrhagic shock, or delayed due to the combination of hypothermia, acidosis and coagulopathy [21]. Unrelenting hemorrhage with no possibility of spontaneous hemostasis results in massive hemoperitoneum. Massive bleeding causes hemodynamic shock and is a predictive factor of death. Hemoperitoneum of venous origin can be contained and tamponnaded by the pressure of the abdominal compartment, which, if released surgically, can result in catastrophic rebleeding and cardiovascular collapse [21].

\subsection{RPH}

Retroperitoneal hematoma occurs in more than $90 \%$ of abdominal vascular injuries. Wounded veins bleed at low pressure into contained spaces and spontaneous hemostasis can be seen. Contained hematomas are not expansive and are more common on the right. RPH of arterial origin is bright red, rapidly expansive and more commonly occurs on the left [21]. A simple classification is to divide RPH into 3 zones (Zone I is the central area from hiatus to sacrum, Zone II is the lateral retroperitoneal area on either side or zone III is the pelvis) (Figure 6) [22].

\subsection{Clinical Diagnosis}

- Major vascular injury should be suspected when the penetrating wound (entry and/or exit) is located between the root of the thigh and the nipples [21]. 


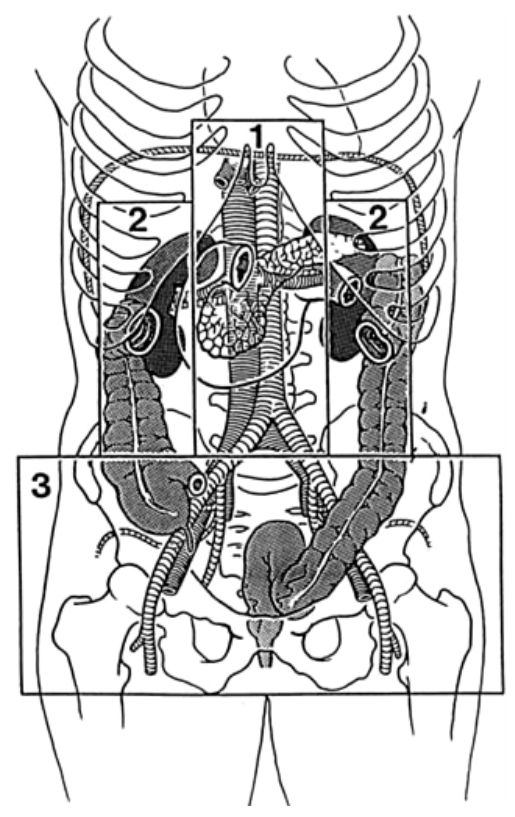

Figure 6. Classification of RPH.

- When the trajectory of the projectile passes through the abdomen and/or the pelvis, or when it is betrayed by externalized bleeding [21].

- Shock out of proportion to the extent of external injury suggests abdominal vascular injury.

- Isolated abdominal injury in patients with shock suggests major vascular injury that requires emergency laparotomy for control [22].

- Abdominal distension in association with signs of acute anemia and/or hemorrhagic shock is a strong indicator of major vascular injury.

- Abdominal wounds near the midline are suggestive of caval or aortic injury; in particular, a periumbilical wound should lead to suspicion of a lesion at the bifurcation of these two vessels.

- Even the slightest hypotension noted in the setting of an abdominal penetrating wound should suggest the possibility of a vascular injury and should lead to surgical intervention unless proven otherwise [21].

\subsection{Work-Up}

Hemodynamic stability determines whether there is time for pre-operative investigations and governs therapeutic choices [21]. The critical patient should undergo immediate exploratory laparotomy [22] whereas the stable patient is to be investigated on leisure. Plain films can reveal pelvic fractures and radio-opaque foreign bodies [22]. Hemoperitoneum is easily diagnosed by bedside color echo Doppler, which can also assess blood flow of the lower extremities. Intravenous pyelograms (IVP) are used to assess the excretory function of each kidney. However, CT scan is the most useful investigation: rapidly performed, it effectively reveals the location of foreign bodies identifies organ damage and evaluates the anatomy of the RPH. One shot intravenous injection of contrast material determines if bleeding is ongoing and evaluates the function and anatomy of the urinary tract. Active bleeding can be controlled with angiography and embolization in some cases. Because modern CT scanners can effectively delineate vascular anatomy, the role of angiography is essentially therapeutic; this invasive diagnostic modality requires adequate equipment and the availability of an experienced operator [21].

\section{Treatment}

\subsection{Surgery}

After the abdomen is entered, immediate control of the supraceliac aorta should be considered before continuing the operation. Retroperitoneal hematomas should not be explored right away unless they are actively bleeding. Stopping the procedure after the initial exploration for damage control to allow time for resuscitation in the in- 
tensive care unit is often a reasonable initial treatment [21] [23]. If the patient's condition allows and if endovascular methods are available, considering placing an aortic balloon from the left brachial artery for temporary occlusion [22].

Several vessels are notoriously difficult to expose. These include the retrohepatic IVC; suprarenal aorta; the celiac axis; the proximal SMA; the junction of the SMV, splenic, and portal veins; and the bifurcation of the vena cava. Maneuvers have been described to aid in the exposure of all of these vessels [22]. Packing is very important for temporary hemorrhage control. Right and left medial visceral rotation, pelvic vascular isolation and Pringle maneuver are few recommended techniques for exposure and control of AVIs [22].

In surgery for AVIs, control of hemorrhage takes priority over avoiding organ ischemia [21]. Ligation is always possible except for the suprarenal aorta and the supra hepatic IVC. Ligation of the portal vein should be avoided as much as possible [21]. Ligation of right renal vein is equivalent to nephrectomy while left renal vein can be ligated [21].

Management of RPH depends upon location: central RPHs should be explored; retrohepatic RPH need packing; perirenal RPH should be explored, unless hematoma is stable in an otherwise unstable patient. RPH in the pelvis is managed by surgical packing and/or endovascular techniques [21].

\subsection{Endovascular Techniques}

These are increasingly used in the acute phase of abdominal trauma. Endovascular treatment is reserved for stable patients, particularly for those with iatrogenic injury, contained RPH, and certain instances of hepatic lesions. This less-invasive alternative can decrease the complexity of surgery. In the unstable patient, endovascular management can complement the surgical gesture, performed before, during or after the operation. In pelvic lesions or in the case of isolated vessel injury, with a contained RPH, this modality can be as effective as surgery [21].

\section{Conclusion}

General surgeons used to manage vascular trauma before the emergence of vascular surgery as a separate specialty from general surgery. There is good evidence in the literature that general surgeons adequately exposed to trauma surgery are able to safely manage extremity vascular trauma achieving limb salvage rate comparable to that obtained by proper vascular surgeons. Abdominal vascular injuries can be encountered in up to one quarter of abdominal trauma. Unless the general surgeon is well prepared, the management of such challenging injuries can be a nightmare. In Iraq, and many other developing countries with limited number of proper vascular surgeons, there is a need to extend the time table allocated for the general surgical trainee in vascular surgery to cope with the increasing terrorism-related casualties.

\section{Acknowledgements}

I wish to thank my lovely daughter Aumnia A Taha for drawing Figure 1.

\section{References}

[1] Vascular Surgery. From Wikipedia, The Free Encyclopedia [Internet Monograph]. http://en.wikipedia.org/wiki/Vascular_surgery

[2] Nikolai Korotkov. From Wikipedia, The Free Encyclopedia [Internet Monograph]. http://en.wikipedia.org/wiki/Nikolai_Korotkov

[3] The Royal College of Surgeons of England. The Surgical Specialties: 10-Vascular Surgery [Internet Monograph]. https://www.rcseng.ac.uk/media/media-background-briefings-and-statistics/the-surgical-specialties-10-vascular-surger y

[4] Taha, A.Y. (2004) Vascular Injuries in Basra, Iraq: A 5-Year Experience. The Journal of the Arab Board of Medical Specializations, 6, 118-125.

[5] Hussain, M.I., Zabid, M., Khan, A., Askri, H. and Khan, A.A. (2009) Extremity Vascular Trauma. A 7-Year Experience in Lahore, Pakistan. Saudi Medical Journal, 30, 50-55.

[6] Taha, A.Y. (2014) Extremity Vascular Injuries [Unpublished Lecture Notes]. School of Medicine, University of Sulaimaniyah. Lecture Given 2014 June 15. https://sulaimaniu.academia.edu/AbdulsalamTaha 
[7] Majid, H.J., Waris, M., Afzal, M.F., Islam, H.R., Khan, S.A., Chaudhry, Z.A., et al. (2000) Extremity Vascular Trauma-The Continuing Challenge. Annals of King Edward Medical College, 6, 137-140.

[8] Frykberg, E.R., Dennis, J.W., Bishop, K., Laneve, L. and Alexander, R.H. (1991) The Reliability of Physical Examination in the Evaluation of Penetrating Extremity Trauma for Vascular Injury: Results at One Year. Journal of Trauma, 31, 502-511. http://dx.doi.org/10.1097/00005373-199104000-00009

[9] Ali, A.A., Gondal, K.M., Khan, A.Z., Aslam, M.N., Butt, T.M. and Chaudhry, A.M. (2001) Factors Influencing the Outcome of Arterial Injuries. Annals of King Edward Medical College, 7, 106-109.

[10] Author (Anonymous) (2015) Clinical Practice Guidelines: Vascular Inuries. Emergency War Surgery [Internet]. http://usaisr.amedd.army.mil/clinical_practice_guidelines.html

[11] Bynoe, R.P., Miles, W.S., Bell, R.M., Greenwold, D.R., Sessions, G., Haynes, J.L., et al. (1991) Noninvasive Diagnosis of Vascular Trauma by Duplex Ultrasonography. Journal of Vascular Surgery, 14, 346-352. http://dx.doi.org/10.1016/0741-5214(91)90087-B

[12] Carrillo, E.H., Spain, D.A., Miller, F.B. and Richardson, J.D. (2002) Femoral Vessel Injuries. Surgical Clinics of North America, 82, 49-65. http://dx.doi.org/10.1016/S0039-6109(03)00140-3

[13] Bowley, D.M., Degiannis, E., Goosen, J. and Boffard, K.D. (2002) Penetrating Vascular Trauma in Johannesburg, South Africa. Surgical Clinics of North America, 82, 221-235. http://dx.doi.org/10.1016/S0039-6109(03)00151-8

[14] Meissner, M., Paun, M. and Johansen, K. (1991) Duplex Scanning for Arterial Trauma. The American Journal of Surgery, 161, 552-555. http://dx.doi.org/10.1016/0002-9610(91)90897-M

[15] Taha, A.Y. (2014) Exposure of Major Blood Vessels (Unpublished Lecture Notes). School of Medicine, University of Sulaimaniyah. Lecture Given 2014 June 15. https://sulaimaniu.academia.edu/AbdulsalamTaha

[16] Taha, A.Y. (2013) Principles of Vascular Anastomosis (Unpublished Lecture Notes). School of Medicine, University of Sulaimaniyah. Lecture Given 2013 July 15. https://sulaimaniu.academia.edu/AbdulsalamTaha

[17] Porter, J.M. and Ivatury, R.R. (2001) Should Trauma Surgeons Render Definitive Vascular Repair in Peripheral Vascular Injuries? The American Surgeon, 67, 427-429.

[18] De Bakey, M.E. and Simeone, F.A. (1946) Battle Injuries of the Arteries in World War II. An Analysis of 2,471 Cases. Annals of Surgery, 123, 534-579. http://dx.doi.org/10.1097/00000658-194604000-00005

[19] Rich, N.M., Baugh, J.H. and Hughes, C.W. (1970) Acute Arterial Injuries in Vietnam: 1,000 Cases. The Journal of Trauma, 10, 359-369. http://dx.doi.org/10.1097/00005373-197005000-00001

[20] Clouse, W.D., Rasmussen, T.E., Peck, M.A., Eliason, J.L., Cox, M.W., Bowser, A.N., et al. (2007) In-Theatre Management of Vascular Surgery: 2 Years of the Balad Vascular Registry. Journal of the American College of Surgeons, 204, 625-632. http://dx.doi.org/10.1016/j.jamcollsurg.2007.01.040

[21] Chapellier, X., Sockeel, P. and Baranger, B. (2010) Management of Penetrating Abdominal Vessel Injuries. Journal of Visceral Surgery, 147, e1-e12. http://dx.doi.org/10.1016/j.jviscsurg.2010.06.003

[22] Taha, A.Y. (2013) Abdominal Vascular Injuries (Unpublished Lecture Notes). School of Medicine, University of Sulaimaniyah. Lecture Given 2013 June 11. https://sulaimaniu.academia.edu/AbdulsalamTaha

[23] Damage Control Surgery. From Wikipedia, the Free Encyclopedia (Internet Monograph).

http://en.wikipedia.org/wiki/Damage control surgery 